\title{
Looking for outflow and infall signatures in high-mass star-forming regions
}

\author{
P. D. Klaassen ${ }^{1,2}$, L. Testi ${ }^{1,3}$, and H. Beuther ${ }^{4}$ \\ ${ }^{1}$ European Southern Observatory, Karl Schwarzschild Str 2, 85748 Garching, Germany \\ e-mail: klaassen@strw.leidenuniv.nl \\ 2 Leiden Observatory, Leiden University, PO Box 9513, 2300 RA, Leiden, The Netherlands \\ ${ }^{3}$ INAF - Osservatorio Astrofisico di Arcetri, Largo E. Fermi 5, 50125 Firenze, Italy \\ ${ }^{4}$ Max Planck Institute for Astronomy, Königstuhl 17, 69117 Heidelberg, Germany
}

Received 28 October 2011 / Accepted 3 December 2011

\section{ABSTRACT}

\begin{abstract}
Context. Many physical parameters change with time in star-forming regions. Here we attempt to correlate changes in the infall and outflow motions in high-mass star-forming regions with evolutionary stage using JCMT observations.

Aims. From a sample of 45 high-mass star-forming regions in three phases of evolution, we investigate the presence of established infall and outflow tracers to determine whether there are any trends attributable to the age of the source.

Methods. We obtained JCMT observations of $\mathrm{HCO}^{+} / \mathrm{H}^{13} \mathrm{CO}^{+} J=4-3$ to trace large-scale infall, and $\mathrm{SiO} J=8-7$ to trace recent outflow activity. We compared the infall and outflow detections to the evolutionary stage of the host source (high-mass protostellar objects, hypercompact HII regions, and ultracompact HII regions).

Results. We find a surprising lack of $\mathrm{SiO}$ detections in the middle stage (hypercompact HII regions), which may be an observational bias. When $\mathrm{SiO}$ is detected, we find that the integrated intensity of the line increases with evolutionary stage. The integrated intensity of $\mathrm{SiO}$ varies with the full width at half maximum of the $\mathrm{H}^{13} \mathrm{CO}^{+}$. All of the sources with infall signatures onto ultracompact $\mathrm{HII}$ regions have corresponding outflow signatures as well.
\end{abstract}

Key words. ISM: molecules - stars: formation - ISM: jets and outflows - submillimeter: ISM - HII regions -

ISM: kinematics and dynamics

\section{Introduction}

High-mass stars are vital players in the evolution of galaxies. During their formation they inject energy into their surroundings in the form of outflows and ionizing photons. As they evolve, their stellar winds continue to stir turbulence in the surrounding medium, and when they explode as supernovae they additionally enhance the metallicity of their environs.

The processes involved in this earliest stage of the formation of high-mass stars are not nearly as well constrained as are the mechanisms responsible for lower mass stars. The premain sequence evolution of low-mass stars (i.e. Shu et al. 1987; André et al. 1993) has been well constrained, and statistically based lifetimes of the stages have developed, in part, from observing large numbers of individual sources (i.e. Enoch et al. 2009; Spezzi et al. 2008).

Similar studies for the early evolution of high-mass stars $\left(M \gtrsim 8 M_{\odot}\right)$ have proven problematic owing to limiting factors, such as the large average distances to high-mass star-forming regions and the clustered nature of their formation. However, studies such as Beltrán et al. (2011) suggest high-mass stars are likely to form though disk-mediated accretion in a similar but scaled up version of lower mass star formation. An evolutionary sequence is developing, but progress is hampered by a lack of large sample surveys of the molecular gas dynamics in these regions (see, for example Zinnecker \& Yorke 2007; Beuther et al. 2007). Evolution in low-mass systems is often characterized by changes in the spectral energy distributions (SEDs). This is not as easily done in high-mass systems (Molinari et al. 2008), which is why studying the gas dynamics is very important.

In broad terms, the evolution of massive protostars begins in an infrared dark cloud (IRDC, i.e. Egan et al. 1998). The central condensation then begins heating its environment, at which point the core starts emitting at IR wavelengths, and it becomes a high-mass protostellar object (HMPO, i.e. Sridharan et al. 2002) within a hot core. The protostar continues to gain mass and heat its environment eventually forming a hypercompact (HC) HII region (i.e. Keto 2007) which then grows into an ultracompact (UC) HII region (i.e. Wood \& Churchwell 1989). This sequence has been shown graphically in Zapata et al. (2010). The goal of this paper is to follow the larger scale (observable with a single dish) outflow and infall structures surrounding sources in the last three of these four evolutionary stages (HMPO, HCHII, and UCHII regions), and determine whether any evolutionary trends are distinguishable.

In the case of low-mass star formation, André et al. (1993) conclude that outflow motions can be seen quite early in the Class 0 phase, and Bontemps et al. (1996) state that the outflows from Class 0 sources are more powerful than those from Class I sources at the same luminosity. They also suggest that all deeply embedded low-mass protostars should have outflows. As for infall in the low-mass regime, inverse P-Cygni profiles indicative of infall have been seen for a decade now (Di Francesco et al. 2001; Zapata et al. 2008; Furuya et al. 2011), and some observations even suggest that infall is seen perpendicular to the outflow direction (Arce \& Sargent 2004). That these trends can 
be applied to higher mass protostars is starting to be explored (i.e. López-Sepulcre et al. 2011).

Here we present $\mathrm{HCO}^{+}$and $\mathrm{H}^{13} \mathrm{CO}^{+}(J=4-3)$ observations to study the bulk infall signatures in high-mass star-forming regions. We also present $\mathrm{SiO}(J=8-7)$ observations to trace recent outflow activity from the same sources. We have collected a sample of 45 high-mass star-forming regions in three evolutionary stages: HMPOs, and sources with HC and UCHII regions. In Sect. 2 we present our observations and in Sect. 3 we present our methodology. In Sect. 4 we present our results, which we discuss in Sect. 5 where we also conclude.

\section{Observations}

Klaassen \& Wilson $(2007,2008)$ observed 24 high-mass starforming regions with HII region in the hypercompact (2) and ultracompact (22) stages of evolution with Receiver B and HARP-B on the James Clerk Maxwell Telescope (JCMT) ${ }^{1}$. They observed $\mathrm{HCO}^{+} / \mathrm{H}^{13} \mathrm{CO}^{+}(J=4-3)$ to detect infall signatures, and $\mathrm{SiO}(J=8-7)$ to trace current outflow motions.

Here we are adding observations of earlier stage sources: 12 HMPOs, and nine hypercompact HII regions in the same tracers as mapped with HARP-B in August 2010. These observations (Project M10BI03) were taken in weather bands 2 and 3 with a goal rms noise level of $0.1 \mathrm{~K}$ in the $T_{\mathrm{A}}^{*}$ temperature scale for the $356 \mathrm{GHz}$ observations $\left(\mathrm{HCO}^{+}\right)$and $0.07 \mathrm{~K}$ for the $347 \mathrm{GHz}$ observations $\left(\mathrm{H}^{13} \mathrm{CO}^{+} / \mathrm{SiO}\right)$. The velocity resolutions were $0.42 \mathrm{~km} \mathrm{~s}^{-1}$ and $0.82 \mathrm{~km} \mathrm{~s}^{-1}$ respectively for the 356 and $347 \mathrm{GHz}$ observations. The beam size is $15^{\prime \prime}$ at these frequencies. Position switched $16 \times 16$ pixel jiggle maps were created for each source, and the rms limits achieved for each map are given in Table 1. The central spectra for each source are shown in Fig. 1. There the $\mathrm{H}^{13} \mathrm{CO}^{+}$intensities have been scaled up by a factor of four, and the $\mathrm{SiO}$ intensities by a factor of 16 .

The new observations were reduced using the JCMT pipeline. For the $347 \mathrm{GHz}$ observations of G213.88-11.84, the system was set to "tracking" to remove an angle offset between multiple scans. Once processed, the datacubes were exported to MIRIAD format for further analysis.

\subsection{Source selection}

The HC and UCHII regions studied in Klaassen \& Wilson (2007, 2008) were selected based on having been part of the (Wood \& Churchwell 1989) or (Kurtz et al. 1994) surveys of HII regions associated with high-mass star formation, and in either Plume et al. (1992) or Hunter (1997) to show they had outflow motions.

To probe earlier stages of high-mass star formation, we selected nine more sources from Kurtz et al. (1994) in which the HII regions were smaller than $0.01 \mathrm{pc}$, which are more characteristic of HCHII regions than ultracompact ones and which were previously known to have outflows. The HII region sizes were determined by taking the observed source diameters from Table 3 of Kurtz et al. (1994) and the distances they give in their Table 2. The HMPOs in this study come from the Sridharan et al. (2002) sample of sources. More specifically, we chose sources that were observed by Beuther et al. (2002b) in CO. For simplicity we excluded sources that had multiple velocity components to remove regions that may have multiple outflows, as

\footnotetext{
1 The James Clerk Maxwell Telescope is operated by The Joint Astronomy Centre on behalf of the Science and Technology Facilities Council of the United Kingdom, The Netherlands Organisation for Scientific Research, and the National Research Council of Canada.
}

well as those with the narrowest line widths, to maximize the likelihood of detecting SiO. In summary, the determining factors for the classification in the threes stages were (i) inclusion in Beuther et al. (2002b) (HMPOs); (ii) HII region smaller than $0.01 \mathrm{pc}$ (HCHII regions); (iii) or HII regions larger than $0.01 \mathrm{pc}$ (UCHII regions).

\section{Methodology}

\subsection{Positions for profiles}

It was noted that for a number of sources (11) in this study, the position of the continuum peak and the position where the optically thin $\mathrm{H}^{13} \mathrm{CO}^{+}$is brightest can be offset from each other even at the $15^{\prime \prime}$ resolution of the JCMT at $350 \mathrm{GHz}$. When present, molecular peaks were generally only $15^{\prime \prime}$ (one beam) from the continuum peak, in random directions. This was also noted for a number of the HMPOs presented in Beuther et al. (2002b) at the $11^{\prime \prime}$ resolution of their IRAM observations.

For those sources with emission maps (all of the new data, and those presented in Klaassen \& Wilson 2008) we are able to compare infall and outflow statistics both at the continuum peak position or the molecular peak position. For the HMPOs, the position of the continuum peak is taken from Table 2 of Beuther et al. (2002a), and for the sources from Wood \& Churchwell (1989) and Kurtz et al. (1994), the continuum peak positions were taken from their Tables 4 and 2 (respectively). Below, if the positions are not explicitly stated, properties are derived from the continuum peak and not the (possibly) offset molecular peak. This is to keep the mapped data consistent with the single pixel data, which was consistently taken at the continuum peak. Whether the molecular line and continuum peaks are offset from each other is noted in the last column of Table 2.

\subsection{Detecting infallsignatures with $\mathrm{HCO}^{+}$}

$\mathrm{HCO}^{+}$is an excellent tracer of high-density gas, like that found in high-mass star-forming regions. It has been used by many authors to trace large-scale infall in both low-mass (Mardones et al. 1997; Gregersen et al. 1997) and high-mass (Fuller et al. 2005; Klaassen \& Wilson 2007, 2008) star-forming regions. CS is another good tracer of high-density gas, however Sun \& Gao (2009) suggest that $\mathrm{HCO}^{+}$is a better tracer of infall motions (see also Tsamis et al. 2008).

The less optically thick $\mathrm{H}^{13} \mathrm{CO}^{+}$isotopologue was also observed in order to distinguish between infall signatures and multiple line of sight components. Double-peaked or asymmetric $\mathrm{HCO}^{+}$line profiles in which the brightest emission is blueward of the source rest velocity can be interpreted as due to infall if the $\mathrm{H}^{13} \mathrm{CO}^{+}$is single peaked at the rest velocity. If the optically thin $\mathrm{H}^{13} \mathrm{CO}^{+}$also has a double-peaked profile, the double peak in both lines is likely due to multiple components along the line of sight. Churchwell et al. (2010) describe the different mechanisms that could produce a double peak in an $\mathrm{HCO}^{+}$line profile towards massive star-forming regions, and conclude that self absorption best fits observed line profiles. The blue or red skew of the absorption profile can then be used to distinguish infall (blue) or outflow (red).

In our study we find 23 sources $(51 \%)$ with blue asymmetries, suggestive of infall in $\mathrm{HCO}^{+} ; 3 / 12$ in the HMPO phase, $8 / 11$ in the HCHII region phase, and 12/22 in the UCHII region phase, see Table 4 and Fig. 2. We define an infall detection for sources with double-peaked $\mathrm{HCO}^{+}$profiles (and single-peaked $\mathrm{H}^{13} \mathrm{CO}^{+}$), such as IRAS18182-1433, and for 
P. D. Klaassen et al.: Infall and outflow in high-mass SFRs

Table 1. Observed sample.

\begin{tabular}{|c|c|c|c|c|c|c|c|}
\hline \multirow[t]{2}{*}{ Name } & \multicolumn{2}{|c|}{ Position (J2000) } & \multicolumn{2}{|c|}{ rms noise limit $(\mathrm{K})$} & \multirow{2}{*}{$\begin{array}{c}V_{\mathrm{LSR}} \\
\left(\mathrm{km} \mathrm{s}^{-1}\right)\end{array}$} & \multicolumn{2}{|c|}{ Distance } \\
\hline & RA & Dec & $347 \mathrm{GHz}$ & $356 \mathrm{GHz}$ & & $(\mathrm{kpc})$ & ref \\
\hline \multicolumn{8}{|c|}{ New Data } \\
\hline G11.11-.4 & 181132 & -193039 & 0.049 & 0.097 & 1 & 5.2 & 4 \\
\hline G213.88-11.84 & 061051 & -061150 & 0.049 & 0.101 & 10 & 5.2 & 4 \\
\hline G033.13-00.09 & 185208 & +000812 & 0.041 & 0.073 & 76.9 & 7.1 & 4 \\
\hline G037.87-00.40 & 190154 & +041249 & 0.040 & 0.074 & 58.8 & 9.2 & 4 \\
\hline G043.24-00.05 & 191034 & +090824 & 0.040 & 0.076 & 7.3 & 11.6 & 4 \\
\hline G048.61+00.02 & 192031 & +135523 & 0.041 & 0.090 & 2.3 & 9.7 & 4 \\
\hline G060.88-00.13 & 194620 & +243529 & 0.042 & 0.102 & 23.3 & 2.3 & 4 \\
\hline G78.438+2.659 & 201939 & +405638 & 0.048 & 0.096 & 3.3 & 3.3 & 4 \\
\hline G081.68+00.54 & 203901 & +421953 & 0.045 & 0.102 & -8.2 & 2 & 4 \\
\hline IRAS05358+3543 & 053915 & +354620 & 0.047 & 0.115 & -16.5 & 1.8 & 3 \\
\hline IRAS18182-1433 & 182110 & -143144 & 0.060 & 0.091 & 59 & 4.5 & 3 \\
\hline IRAS $18264-1152$ & 182915 & -115018 & 0.045 & 0.088 & 44.5 & 3.5 & 3 \\
\hline IRAS $18566+0408$ & 185910 & +041214 & 0.060 & 0.074 & 86.5 & 6.7 & 3 \\
\hline IRAS $19035+0641$ & 190602 & +064639 & 0.039 & 0.077 & 32.5 & 2.2 & 3 \\
\hline IRAS $19217+1651$ & 192359 & +165750 & 0.041 & 0.089 & 3 & 10.5 & 3 \\
\hline IRAS $19411+2306$ & 194318 & +231359 & 0.042 & 0.090 & 30 & 2.9 & 3 \\
\hline IRAS20126+4107 & 202324 & +411736 & 0.050 & 0.068 & -1.5 & 1.7 & 3 \\
\hline IRAS22134+5834 & 221509 & +584909 & 0.050 & 0.072 & 18.5 & 2.6 & 3 \\
\hline IRAS23033+5951 & 230525 & +600808 & 0.052 & 0.076 & -53.5 & 3.5 & 3 \\
\hline IRAS23139+5939 & 231611 & +595533 & 0.048 & 0.080 & -43 & 4.8 & 3 \\
\hline IRAS23151+5912 & 231721 & +592849 & 0.061 & 0.071 & -56 & 5.7 & 3 \\
\hline \multicolumn{8}{|c|}{ Klaassen \& Wilson (2008) - maps } \\
\hline G5.89-0.39 & 180030.5 & -240358.00 & 0.238 & 0.070 & 9 & 2 & 2 \\
\hline $\mathrm{G} 10.47+0.03$ & 180838.4 & -195152.00 & 0.082 & 0.074 & 67 & 10.8 & 2 \\
\hline G10.6-0.4 & 181028.0 & -195604.00 & 0.102 & 0.079 & -3 & 6 & 2 \\
\hline G19.61-0.23 & 182738.3 & -115640.00 & 0.090 & 0.098 & 43 & 4.5 & 2 \\
\hline G20.08-0.14 & 182810.7 & -112848.00 & 0.084 & 0.071 & 42 & 4.1 & 2 \\
\hline G29.96-0.02 & 184604.1 & -023922.00 & 0.126 & 0.144 & 98 & 9 & 2 \\
\hline $\mathrm{K} 3-50 \mathrm{~A}$ & 200145.8 & +333242.00 & 0.089 & 0.066 & 0.24 & 8.6 & 2 \\
\hline \multicolumn{8}{|c|}{ Klaassen \& Wilson (2007) - single pointing } \\
\hline G5.97 & 180340.4 & -242244 & 0.044 & 0.069 & 10 & 2.7 & 1 \\
\hline G8.67 & 180619.0 & -213732 & 0.068 & 0.074 & 36 & 8.5 & 1 \\
\hline G12.21 & 181239.7 & -182421 & 0.042 & 0.076 & 24 & 16.3 & 1 \\
\hline M17S & 182024.8 & -161135 & 0.044 & 0.077 & 20 & 2.3 & 1 \\
\hline G31.41 & 184733.0 & -011236 & 0.044 & 0.061 & 97 & 8.5 & 1 \\
\hline G34.26 & 185318.5 & 011458 & 0.047 & 0.131 & 58 & 3.7 & 1 \\
\hline G45.07-0.13 & 191322.1 & 105053 & 0.044 & 0.073 & 59 & 9.7 & 1 \\
\hline G45.47 & 191425.6 & 110926 & 0.037 & 0.063 & 58 & 8.3 & 1 \\
\hline G61.48 & 194649.2 & 251248 & 0.037 & 0.048 & 12 & 2 & 1 \\
\hline G75.78 & 202144.1 & 372640 & 0.038 & 0.068 & 0 & 5.6 & 1 \\
\hline Cep A & 225617.9 & 620149 & 0.031 & 0.076 & -10 & 0.7 & 1 \\
\hline $\mathrm{W} 3(\mathrm{OH})$ & 022703.8 & 615225 & 0.026 & 0.079 & -48 & 2.4 & 1 \\
\hline G138.3 & 030129.2 & 602912 & 0.066 & 0.073 & -38 & 3.8 & 1 \\
\hline G139.9 & 030723.9 & 583053 & 0.058 & 0.071 & -39 & 4.2 & 1 \\
\hline G192.58 & 061253.6 & 175927 & 0.037 & 0.053 & 9 & 2.5 & 1 \\
\hline G192.6 & 061253.6 & 180026 & 0.074 & 0.098 & 9 & 2.5 & 1 \\
\hline G240.3 & 074451.9 & -240740 & 0.035 & 0.048 & 68 & 6.4 & 1 \\
\hline
\end{tabular}

References. Distances are given in: (1) Klaassen \& Wilson (2007), (2) Klaassen \& Wilson (2008), (3) Sridharan et al. (2002), and (4) Kurtz et al. (1994).

sources with asymmetrically blue profiles containing red shoulders like G81.68. Of the 14 sources in the mapped data with infall signatures 6(7) of these sources have double-peaked profiles at the continuum (molecular) peak positions. For all sources with double-peaked $\mathrm{HCO}^{+}$profiles with stronger blue peaks than red, we were able to calculate infall velocities and mass infall rates. We determined the velocities and intensities of the blue and red peaks and that of the absorption feature at its minimum. We also determined the FWHM of the optically thin $\mathrm{H}^{13} \mathrm{CO}^{+}$and input all of these quantities into Eq. (9) of Myers et al. (1996) to determine the infall velocity. To determine the mass infall rate, we used Eq. (3) of Klaassen \& Wilson (2007) where $\dot{M}=$ $(4 / 3) \pi n_{\mathrm{H}_{2}} \mu m_{\mathrm{H}} r^{2} V_{\text {in }}$ is the mass infall rate, $\mu$ the mean molecular weight (2.35), $m_{\mathrm{H}}$ the mass of Hydrogen, $r$ the radius of the emitting region (assumed here to be the beam radius), $V_{\text {in }}$ the infall velocity, and $n_{\mathrm{H}_{2}}$ the ambient density taken from previous studies (i.e. Beuther et al. 2002a; Plume et al. 1997; Shirley et al. 2003). For sources where no ambient density was found, the average of the ambient densities from the other sources $\left(n_{\mathrm{H}_{2}}=10^{6}\right)$ was used. These values are shown in Table 3. For sources with 

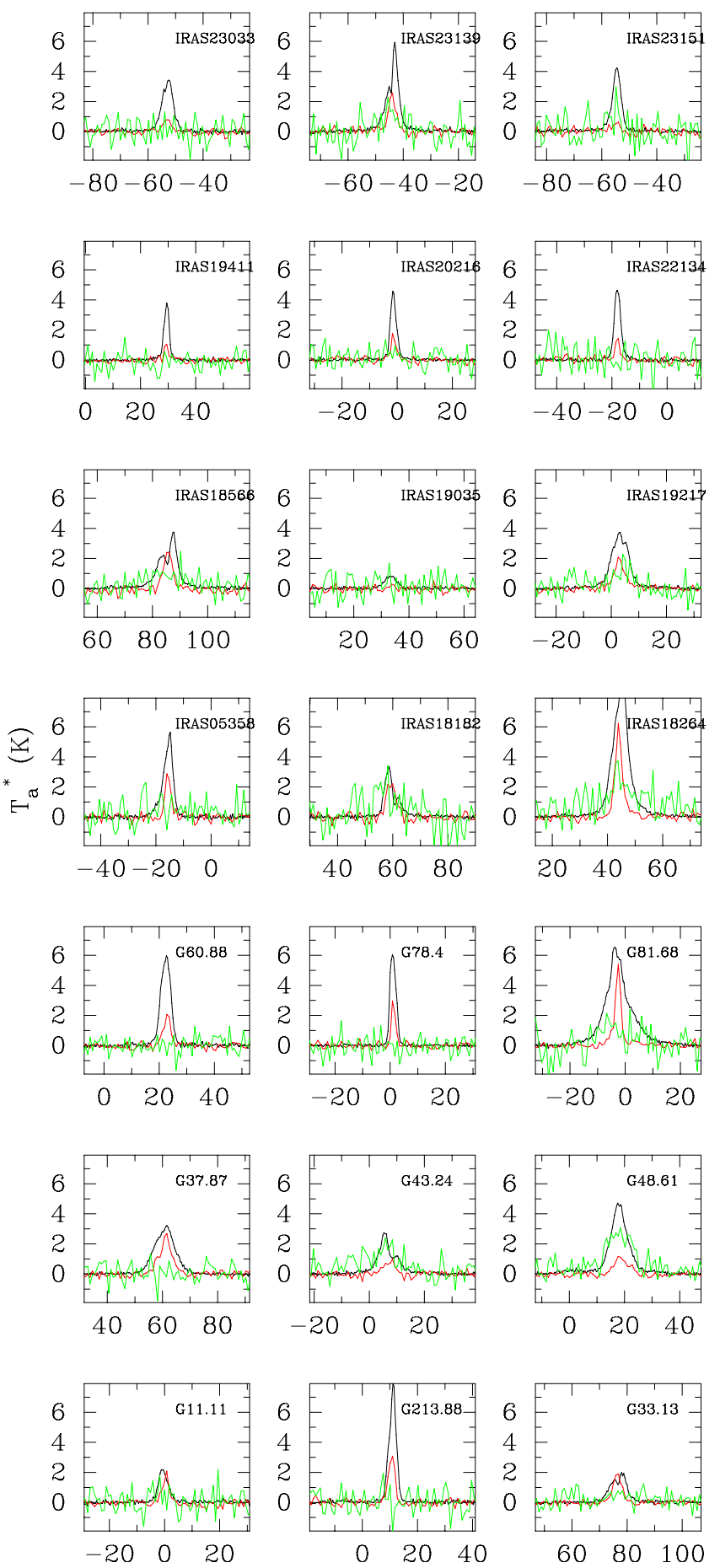

$$
\mathrm{V}_{\mathrm{lsr}}\left(\mathrm{km} \mathrm{s}^{-1}\right)
$$

Fig. 1. Spectra from new sources. Spectra of previously published sources can be found in Klaassen \& Wilson (2007) and Klaassen \& Wilson (2008). The scale on the $Y$ axis reflects the intensity of the $\mathrm{HCO}^{+}$line(black). The $\mathrm{H}^{13} \mathrm{CO}^{+}$(red) has been scaled up by a factor of four, and the $\mathrm{SiO}$ (green) has been scaled up by a factor of 16 .

a "shoulder" infall signature, we were unable to calculate these properties. As the mass infall rate scales with the square of the infalling radius, the effects of beam dilution (source smaller than the beam) are likely to be overestimating the mass infall rate.

Our HMPOs are drawn from the same sample as those of Fuller et al. (2005). Of our 12 HMPOs, eight overlap with those in their sample that have significant line asymmetries
Table 2. Infall and outflow detections.

\begin{tabular}{|c|c|c|c|c|c|}
\hline Source & Type & Infall & Outflow & Offset & Notes \\
\hline IRAS05358+3543 & HMPO & $\mathrm{N}$ & $\mathrm{CM}$ & both & $2-1$ \\
\hline IRAS18182-1433 & HMPO & $\mathrm{CM}$ & $\mathrm{CM}$ & both & $2-1$ \\
\hline IRAS18264-1152 & HMPO & $\mathrm{N}$ & $\mathrm{CM}$ & & $2-1$ \\
\hline IRAS18566+0408 & HMPO & $\mathrm{N}$ & $\mathrm{CM}$ & & $2-1$ \\
\hline IRAS19035+0641 & HMPO & $\mathrm{N}$ & $\mathrm{N}$ & both & $2-1$ \\
\hline IRAS19217+1651 & HMPO & $\mathrm{N}$ & $\mathrm{CM}$ & & $2-1$ \\
\hline IRAS19411+2306 & HMPO & $\mathrm{N}$ & $\mathrm{N}$ & & $2-1$ \\
\hline IRAS20126+4107 & HMPO & $\mathrm{N}$ & $\mathrm{N}$ & 356 & $2-1$ \\
\hline IRAS22134+5834 & HMPO & $\mathrm{CM}$ & $\mathrm{N}$ & both & \\
\hline IRAS23033+5951 & HMPO & $\mathrm{N}$ & $\mathrm{CM}$ & both & $2-1$ \\
\hline IRAS23139+5939 & HMPO & $\mathrm{N}$ & M & & $2-1$ \\
\hline IRAS23151+5912 & HMPO & $\mathrm{CM}$ & $\mathrm{N}$ & 347 & $2-1$ \\
\hline G11.11-0.4 & HCHII & $\mathrm{CM}$ & $\mathrm{N}$ & & \\
\hline G213.88-11.84 & HCHII & $\mathrm{M}$ & M & both & \\
\hline G033.13-00.09 & HCHII & $\mathrm{N}$ & $\mathrm{N}$ & & \\
\hline G037.87-00.40 & HCHII & $\mathrm{C}$ & $\mathrm{N}$ & & \\
\hline G043.24-00.05 & HCHII & $\mathrm{CM}$ & $\mathrm{CM}$ & & \\
\hline $\mathrm{G} 048.61+00.02$ & $\mathrm{HCHII}$ & $\mathrm{N}$ & $\mathrm{CM}$ & & \\
\hline G060.88-00.13 & $\mathrm{HCHII}$ & $\mathrm{CM}$ & $\mathrm{N}$ & both & \\
\hline $\mathrm{G} 78.438+2.659$ & HCHII & M & $\mathrm{N}$ & 347 & \\
\hline G081.68+00.54 & HCHII & $\mathrm{CM}$ & M & both & \\
\hline M17S & HCHII & Y & $\mathrm{N}$ & & $1 \mathrm{p}$ \\
\hline G61.48 & HCHII & $\mathrm{N}$ & $\mathrm{N}$ & & $1 \mathrm{p}$ \\
\hline G5.89 & UCHII & $\mathrm{N}$ & $\mathrm{CM}$ & & \\
\hline G10.47 & UCHII & $\mathrm{CM}$ & $\mathrm{CM}$ & both & \\
\hline G10.6 & UCHII & $\mathrm{CM}$ & $\mathrm{CM}$ & both & \\
\hline G19.61 & UCHII & $\mathrm{CM}$ & $\mathrm{CM}$ & both & \\
\hline G20.08 & UCHII & $\mathrm{CM}$ & M & both & \\
\hline G29.962 & UCHII & $\mathrm{N}$ & $\mathrm{CM}$ & both & \\
\hline K3-50A & UCHII & $\mathrm{N}$ & $\mathrm{CM}$ & both & \\
\hline G5.97 & UCHII & $\mathrm{N}$ & $\mathrm{N}$ & & $1 \mathrm{p}$ \\
\hline G8.67 & UCHII & Y & Y & & $1 \mathrm{p}$ \\
\hline G12.21 & UCHII & $\mathrm{N}$ & Y & & $1 \mathrm{p}$ \\
\hline G31.41 & UCHII & Y & $\mathrm{N}$ & & $1 \mathrm{p}$ \\
\hline G34.26 & UCHII & Y & Y & & $1 \mathrm{p}$ \\
\hline G45.073 & UCHII & $\mathrm{N}$ & Y & & $1 \mathrm{p}$ \\
\hline G45.47 & UCHII & $\mathrm{N}$ & $\mathrm{Y}$ & & $1 \mathrm{p}$ \\
\hline G75.78 & UCHII & $\mathrm{N}$ & Y & & $1 \mathrm{p}$ \\
\hline Cep A & UCHII & Y & Y & & $1 \mathrm{p}$ \\
\hline $\mathrm{W} 3(\mathrm{OH})$ & UCHII & Y & Y & & $1 \mathrm{p}$ \\
\hline G138.3 & UCHII & $\mathrm{N}$ & $\mathrm{N}$ & & $1 \mathrm{p}$ \\
\hline G139.9 & UCHII & $\mathrm{N}$ & $\mathrm{N}$ & & $1 \mathrm{p}$ \\
\hline G192.58 & UCHII & Y & Y & & $1 \mathrm{p}$ \\
\hline G192.6 & UCHII & Y & $\mathrm{N}$ & & $1 \mathrm{p}$ \\
\hline G240.3 & UCHII & Y & $\mathrm{N}$ & & $1 \mathrm{p}$ \\
\hline
\end{tabular}

Notes. For those sources with offsets between the continuum and molecular peaks, $\mathrm{C}$ indicates a detection at the continuum peak, and $\mathrm{M}$ indicates a detection at the molecular peak. Column 5 indicates whether there was a shift between the continuum peak and the molecular peak. (356) indicates an offset in the $356 \mathrm{GHz}$ observations, (347) indicates an offset in the $347 \mathrm{GHz}$ observations, and (both) indicates an offset in both tunings. Column 6 notes: $(1 \mathrm{p})-$ Single pointing observations. $(2-1)-\mathrm{SiO} J=2-1$ detection for the HMPOs.

(those listed in their Table 9). In each case, our $\mathrm{HCO}^{+}$line asymmetries are consistent with theirs, save for one source, IRAS22134+5834, where we detect shoulder emission. In the $J=1-0$ and $J=3-2$ transitions of $\mathrm{HCO}^{+}$for this source, they report red asymmetries, and in $J=4-3$ they do not find any significant line asymmetry. They also note that the $\mathrm{H}_{2} \mathrm{CO}$ towards this source shows a blue asymmetry. In their total sample of 78 sources, they suggest 22 are infall candidates. Their detection rate of $28 \%$ is consistent with our detection rate of $25 \%$ in 12 sources. 
P. D. Klaassen et al.: Infall and outflow in high-mass SFRs

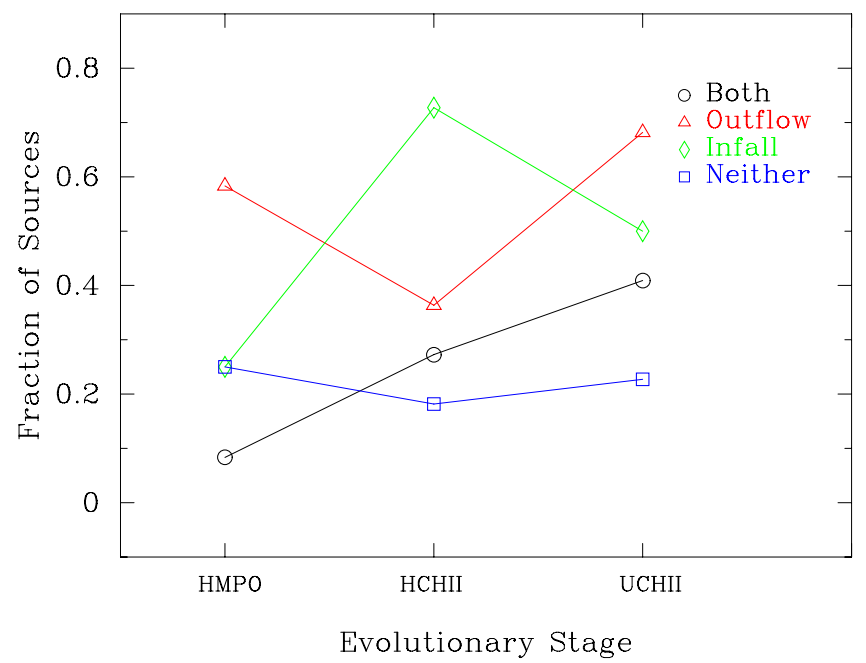

Fig. 2. Fraction of sources in each evolutionary bin with either infall $(\diamond)$, outflow $(\triangle)$, both $(\circ)$, or neither $(\square)$ signature detected. Here, any source with infall or outflow is plotted with their respective datapoint, as well as with "both" labels, as appropriate. Plotting this way shows that there is strong evidence for ongoing infall and outflow during the later stages of evolution.

Table 3. Infall rates.

\begin{tabular}{|c|c|c|c|c|}
\hline \multirow[t]{2}{*}{ Source } & \multicolumn{2}{|c|}{ Continuum peak } & \multicolumn{2}{|c|}{ Molecular peak } \\
\hline & $\begin{array}{c}V_{\text {in }} \\
\mathrm{km} \mathrm{s}^{-1}\end{array}$ & $\begin{array}{c}\dot{M}_{\mathrm{in}} \\
10^{-2} \dot{M}_{\mathrm{yr}^{-1}}\end{array}$ & $\begin{array}{c}V_{\text {in }} \\
\mathrm{km} \mathrm{s}^{-1}\end{array}$ & $\begin{array}{c}\dot{M}_{\text {in }} \\
10^{-2} \dot{M}_{y^{-1}}\end{array}$ \\
\hline IRAS18182 & $1.74 \pm 0.58$ & $1.62 \pm 0.54$ & $1.57 \pm 0.56$ & $1.46 \pm 0.52$ \\
\hline G43.24 & $1.27 \pm 0.33$ & $5.62 \pm 1.44$ & $1.61 \pm 0.45$ & $7.12 \pm 2.01$ \\
\hline G81.68 & \multicolumn{2}{|c|}{ Shoulder } & $0.26 \pm 0.07$ & $0.036 \pm 0.01$ \\
\hline G10.47 & \multirow{2}{*}{\multicolumn{2}{|c|}{$\begin{array}{l}\text { same as mol. peak } \\
\text { same as mol peak }\end{array}$}} & $0.42 \pm 0.11$ & $1.6 \pm 0.43$ \\
\hline G10.6 & & & $0.05 \pm 0.03$ & $0.06 \pm 0.03$ \\
\hline G & \multicolumn{2}{|c|}{ same as mol. peak } & $0.21 \pm 0.08$ & $0.14 \pm 0.05$ \\
\hline G20.08 & \multicolumn{2}{|c|}{ same as mol. peak } & $0.05 \pm 0.03$ & $0.03 \pm 0.02$ \\
\hline G8.67 & $0.4 \pm 0.1$ & $0.04 \pm 0.02$ & \multicolumn{2}{|c|}{ Single Pointing } \\
\hline M17S & $1.4 \pm 0.5$ & $0.04 \pm 0.02$ & \multicolumn{2}{|c|}{ Single Pointing } \\
\hline Cep A & $0.23 \pm 0.07$ & $0.002 \pm 0.001$ & \multicolumn{2}{|c|}{ Single Pointing } \\
\hline W3 $(\mathrm{OH})$ & $0.06 \pm 0.02$ & $0.03 \pm 0.01$ & \multicolumn{2}{|c|}{ Single Pointing } \\
\hline G192.58 & $0.8 \pm 0.3$ & no data & \multicolumn{2}{|c|}{ Single Pointing } \\
\hline G192.6 & $0.9 \pm 0.4$ & $0.02 \pm 0.01$ & \multicolumn{2}{|c|}{ Single Pointing } \\
\hline G34.26 & $1.5 \pm 0.3$ & $0.14 \pm 0.04$ & Single & Pointing \\
\hline
\end{tabular}

Notes. Single lines separate the three stages (HMPO, HCHII, and UCHII regions from top to bottom). Velocities are in units of $\mathrm{km} \mathrm{s}^{-1}$ and mass infall rates in units of $10^{-2} M_{\odot} \mathrm{yr}^{-1}$. Infall velocities derived for UCHII regions are given in Klaassen \& Wilson $(2007,2008)$.

Twelve of our sources overlap with the HCO+ $(J=3-2)$ study of Reiter et al. (2011). For 11 of these sources, our line profiles (blue vs. red asymmetry) are consistent. For the other source (CepA), we find that our line profiles are consistent with the HCN profiles shown in $\mathrm{Wu}$ et al. (2010) and $\mathrm{Wu} \&$ Evans (2003) and the $\mathrm{HCO}^{+}(J=1-0)$ profile in Sun \& Gao (2009). Reiter et al. (2011) suggest that there is outflow contamination in their 30" beam for CepA.

The study of Churchwell et al. (2010) observed a number of $\mathrm{HII}$ regions in $\mathrm{HCO}^{+}(J=3-2,2-1)$. Nine of their sources overlap with our study. We find that for all nine sources, whether or not we detect an infall signature is consistent with their findings. This includes G33.13, where we both find red-peaked profiles (which indicates outflow). They note that of their 24 HII regions (both ultracompact and hyper compact), they only detect infall
Table 4. Infall and outflow statistics.

\begin{tabular}{lccccc}
\hline \hline Stage & \# of sources & Infall & Outflow & Both & Neither \\
\hline HMPO & 12 & 3 & 7 & 1 & 4 \\
HCHII & 11 & 8 & 4 & 3 & 2 \\
UCHII & 22 & 12 & 16 & 9 & 5 \\
\hline
\end{tabular}

Notes. Fraction of infall and outflow detections as a function of evolutionary stage.

signatures in eight sources (33\%). For our 33 HII regions, we detect infall in $20(61 \%)$.

There are also nine sources in our survey that overlap with the study of López-Sepulcre et al. (2010). We compared our infall detections with their detections in $\mathrm{HCO}^{+} J=1-0$, and find that we agree for six out of nine sources. The sources for which we disagree are G192.58, G192.6 and IRAS23151. For each of these three sources, we detect infall in regions where they do not. We suggest that our detections are partly due to our higher resolution (15" vs. 29"), and because at earlier times, the $J=4-3$ transition is likely a better asymmetry tracer than $J=1-0$ (see Fig. 8 of Tsamis et al. 2008). This statement is supported by comparison with the $\mathrm{HCO}^{+} J=1-0$ observations of Purcell et al. (2006). Many more of their $\mathrm{HCO}^{+}$lines were double-peaked, and these asymmetries are equally split between red and blue. Our asymmetries are skewed towards blue asymmetries. The higher energy transition does not self absorb as readily, and stronger velocity gradients may be required to show an infall signature.

\subsection{Detecting outflow signatures with $\mathrm{SiO}$}

As stated in Sect. 2, all of the sources in this survey were selected based on previous outflow signatures having been detected (in, for instance $\mathrm{CO}$ or $\mathrm{CS}$ ). Here, to classify sources as having active outflows, we require a detection of $\mathrm{SiO} J=8-7$. $\mathrm{SiO}$ is formed in shocked regions as $\mathrm{Si}$ is liberated from dust grains and joins with O (Schilke et al. 1997; Caselli et al. 1997). The Si then evolves out of species observable from the ground in $\sim 10^{4} \mathrm{yr}$ (Pineau des Forets et al. 1997), and therefore $\mathrm{SiO}$ is an excellent tracer of recent shock activity. Because $\mathrm{CO}$ is a bulk gas tracer, and not a specific shock tracer like $\mathrm{SiO}$, it gives a broader sense of the outflow history of the region. Because $\mathrm{SiO}$ is short lived, it traces active outflows.

To systematically determine whether $\mathrm{SiO}$ is detected we determined the width of the $\mathrm{HCO}^{+}$line at the $3 \sigma$ level. This width and these velocity limits were used to create a zeroth-moment map of $\mathrm{SiO}$. If the intensity at the peak pixel (whether continuum or molecular peak) was greater than $3 \sigma$ in that velocity interval, we claimed an $\mathrm{SiO}$ detection.

$\mathrm{SiO}$ was detected at the continuum peak in 23 sources; six in the HMPO phase, two in the HCHII region phase, and 15 in the UCHII region phase (see Table 4 and Fig. 2). For sources with maps, where we could distinguish between the continuum and molecular peak, we found four more detections (1 HMPO, $2 \mathrm{HCHII}$, and $1 \mathrm{UCHII}$ region), bringing the total number of sources with $\mathrm{SiO}$ detections to $27(60 \%)$. In no source where $\mathrm{SiO}$ was detected at the continuum peak was the signal lost at the molecular peak.

We note that the $\mathrm{SiO}$ detection rate for the $J=2-1$ and $J=3-2$ observations of López-Sepulcre et al. (2011) are much higher (88\%). The sources in López-Sepulcre et al. (2011) were 
taken from Rathborne et al. (2006), Beuther et al. (2002b), Faúndez et al. (2004), Hill et al. (2005), Hofner \& Churchwell (1996), Walsh et al. (1998), and Wood \& Churchwell (1989). Reclassifying their IR lound and dark sources into our nomenclature, the Rathborne et al. (2006) sources became Infrared Dark Clouds (IRDCs), The Beuther et al. (2002b) and Faúndez et al. (2004) sources became HMPOs, and the remaining sources were classified as either HC or UCHII regions depending on the size of the HII region taken from the literature. Without taking the excitation of the different transitions or the different beam sizes of the observations into consideration, but purely looking at whether these 2-1 and 3-2 detections would have been made at the sensitivity limits of our observations, we find that only $55-60 \%$ of their sources would be detected in our survey. We are thus likely to be missing a few detections due to our sensitivity limits. We note that by increasing their rms levels to match ours, the fractional detection of $\mathrm{SiO}$ in $\mathrm{HCHII}$ regions drops much lower than it does for the HMPOs and the UCHII regions. This suggests that perhaps our lack of detection of $\mathrm{SiO}$ in HCHII regions may be an observational bias (see Fig. 4).

With regards to the excitation of $\mathrm{SiO} J=8-7$ in our sources, we find very few sources with extended $\mathrm{SiO}$ emission. We compared our $\mathrm{SiO}$ integrated intensities to those of the $J=2-1$ and $J=3-2$ transitions observed in López-Sepulcre et al. (2011). We find a line ratio $(8-7) /(3-2)<1$ for all sources in both surveys that are detected in $\mathrm{SiO}$. Comparing this result to the models of Gusdorf et al. (2008), assuming an ambient density of $10^{6} \mathrm{~cm}^{-3}$, we suggest low shock velocities $\left(<30 \mathrm{~km} \mathrm{~s}^{-1}\right)$ in these sources. As in Leurini et al. (2011), we find that SiO shock models developed for low-mass star-forming regions are consistent with our high-mass star-forming region results as well.

\section{Results}

In Fig. 3 we present a number of source properties averaged by the presumed evolutionary stage of the source. These properties include the FWHM of the $\mathrm{H}^{13} \mathrm{CO}^{+}$, the distance to the sources, the far infrared luminosity of the source, and the integrated intensity of the $\mathrm{SiO}$ lines. Prior to averaging, G5.89 was removed from the sample, because the properties of this source are much greater than $3 \sigma$ away from the averages and can skew our results.

The FWHM of the $\mathrm{H}^{13} \mathrm{CO}^{+}$, a measure of the turbulence in our observed regions, does not seem to correlate with the source evolutionary stage $\left([\right.$ stage $\left.]=0.5 \pm 0.9\left[\mathrm{H}^{13} \mathrm{CO}^{+} \mathrm{FWHM}\right]+3 \pm 2\right)$. The averaged trend in Fig. 3 suggests a possibly weak correlation, but the correlation coefficient is 0.025 when the data is not averaged (as stated in the figure caption). This suggests that the FWHM can be used for determining source properties independent of its age.

We do appear to have a slight distance bias in our survey sources, with older sources being, on average, slightly further away. The correlation coefficient of the averaged data is 0.99 , while the coefficient of the unaveraged data is only 0.25 . The distance differences are not likely to be significant in our analysis ([stage $]=1 \pm 2[\mathrm{D}]+2 \pm 4)$.

The FIR luminosities of our sources, taken from Wood \& Churchwell (1989); Kurtz et al. (1994); Beuther et al. (2002b); Mateen et al. (2006); Walsh et al. (1997) are the IR fluxes from IRAS converted into total luminosities for the sources at the distances listed in those papers. The FIR luminosity can be taken as a tracer of the total luminosity of the source if the distance is known (Wood \& Churchwell 1989). Thus, the FIR luminosity can be used to determine the spectral type of the embedded

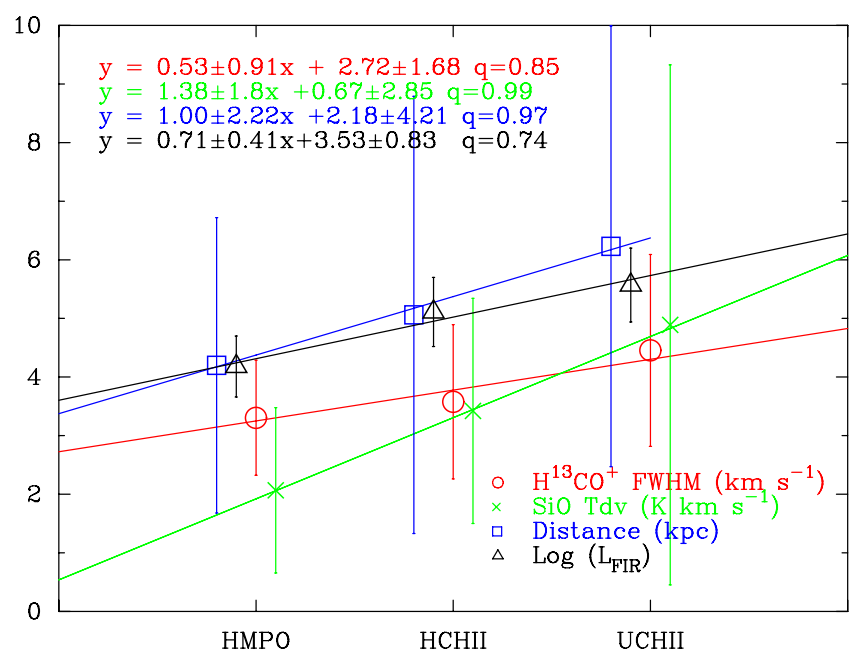

Fig. 3. Source properties averaged over evolutionary stage. The correlation coefficients for the unaveraged properties are $0.025,0.25,0.71$, and 0.35 for the $\mathrm{H}^{13} \mathrm{CO}^{+}$FWHM, Distance, FIR luminosity, and $\mathrm{SiO}$ integrated intensity (respectively).

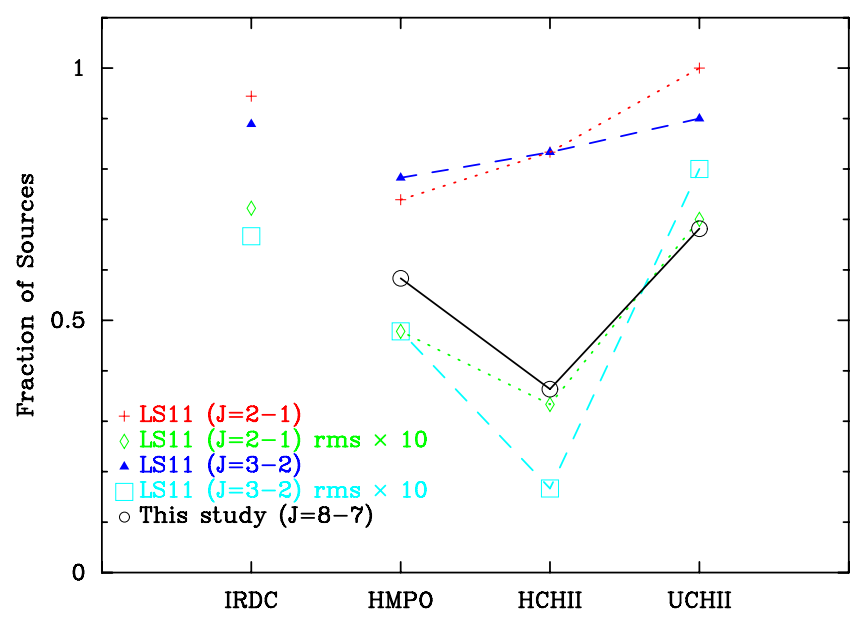

Fig. 4. Comparison between the detection rate of $\mathrm{SiO}$ presented here with that of López-Sepulcre et al. (2011). The short dashed lines represent their $J=2-1$ detections, and the long dashed lines represent their $J=3-2$ detections. The $\diamond$ and $\square$ symbols show the López-Sepulcre et al. (2011) detections corresponding to degrading their sensitivity by a factor of 10 to match our sensitivity. There is a corresponding lack of $\mathrm{HCHII}$ region $\mathrm{SiO}$ detections in all three tracers. This plot does not consider the differences in beam sizes or excitation conditions required for the 3 different $\mathrm{SiO}$ transitions. López-Sepulcre et al. (2011) contained sources at an earlier evolutionary stage than probed here (from the study of Rathborne et al. 2006), and those sources have been included here for completeness.

protostar. On average, the FIR luminosity does not seem to correlate with evolutionary stage $\left([\right.$ stage $]=0.71 \pm 0.41\left[\log L_{\mathrm{FIR}}\right]+$ $3.35 \pm 0.83)$, with a correlation coefficient of 0.74 for three points. When the individual FIR luminosities are considered (and not the average for the stage), the coefficient drops to 0.36. The slope and intercept of the least squares fits to the data remain the same between the averaged and un-averaged data. We thus suggest that the FIR luminosity does not correlate with evolutionary stage. Neither the SIO integrated intensity nor the $\mathrm{H}^{13} \mathrm{CO}^{+}$FWHM correlate with the FIR luminosity. The scaling relation for SIO integrated intensity $(y)$ vs. FIR luminosity $(x)$ is $y=1.6 \pm 0.8 x-4 \pm 4$, while for $\mathrm{H}^{13} \mathrm{CO}^{+}(y)$ vs. FIR luminosity $(x)$ is $y=0.9 \pm 0.2 x-0.7 \pm 1.2$ (with correlation 
Table 5. Source property averages by evolutionary stage.

\begin{tabular}{|c|c|c|c|c|}
\hline Stage & $\begin{array}{c}\mathrm{H}^{13} \mathrm{CO}^{+} \mathrm{FWHM} \\
\left(\mathrm{km} \mathrm{s}^{-1}\right)\end{array}$ & $\begin{array}{l}\mathrm{SiO} \int T \mathrm{~d} v \\
\left(\mathrm{~K} \mathrm{~km} \mathrm{~s}^{-1}\right)\end{array}$ & $\begin{array}{l}\text { Distance } \\
(\mathrm{kpc})\end{array}$ & $\log \left(L_{\mathrm{FIR}}\right)$ \\
\hline HMPO & $3.305 \pm 0.982$ & $2.067 \pm 1.410$ & $4.20 \pm 2.52$ & $4.18 \pm 0.52$ \\
\hline HCHII & $3.578 \pm 1.316$ & $3.422 \pm 1.922$ & $5.06 \pm 3.73$ & $5.11 \pm 0.59$ \\
\hline UCHII & $4.454 \pm 1.636$ & $4.890 \pm 4.436$ & $6.23 \pm 3.76$ & $5.57 \pm 0.62$ \\
\hline $\begin{array}{c}\text { slope } \\
q^{a}\end{array}$ & $\begin{array}{l} \pm 0.9 \\
085\end{array}$ & $\begin{array}{lcl}1.4 \pm & 1.8\end{array}$ & $\begin{array}{lcc}1 & \pm & 2 \\
& 97\end{array}$ & $0.7 \pm 0.4$ \\
\hline
\end{tabular}

Notes. Source properties averaged over evolutionary stage. The unceratinties reflect the standard deviation in the averaged quantities. ${ }^{(a)}$ Correlation coefficient .

coefficients of 0.36 and 0.5 , respectively), nor does the $\mathrm{SiO}$ integrated intensity correlate with distance to source (correlation coefficient of 0.3 ). However, the $\mathrm{H}^{13} \mathrm{CO}^{+}$line width does correlate with distance, as described further in Sect. 4.2.

\subsection{Infall}

We find that our detections of infall signatures drastically changes from the HMPO stage (where we only detect infall in $25 \%$ of our sources) to the HCHII region phase, where we detect infall in $72 \%$ of sources. This result is surprising given that the younger sources should be actively accreting material onto the central source. However, if we consider that the infall may start at the center of the clump and the region undergoing infall expands outwards, then the infall area for a younger source is smaller, and thus more likely to be beam diluted. Studies of the line asymmetry arising from infall (i.e. De Vries \& Myers 2005; Tsamis et al. 2008) suggest that infall signatures are easiest to distinguish when the infall region is best matched to the observing beam. For the HMPOs in our sample, the infall signatures are not preferentially seen in the closer sources. The average distance to a source with an infall detection is $4.6 \mathrm{kpc}$, while the average distance to sources without is $4.1 \mathrm{kpc}$. Thus, we suggest that the lack of detections is not a distance bias, but based on a general beam dilution of the signal.

Assuming the infall is occurring from the inside outward, then in the earlier stages, the infall region is much smaller than our observing beam, and thus the signal is beam diluted. By the time an HCHII region has formed, the infalling region has grown. We therefore have a much higher infall detection rate because the infalling area is much better matched to our beam area. For the UCHII regions, the infall detection rate drops to $50 \%$. As shown above, the $\mathrm{HCO}^{+}$beam filling factor has drastically increased for these sources, and the region undergoing infall should be easier to detect. That our detection rate is not as high may suggest that the outflows in the later stages have begun reversing the large-scale infall, and some of these regions are reaching their final masses.

\subsection{Outflow}

$\mathrm{SiO}$ can be produced in either shocked gas from an outflow or from a photodissociation region (PDR) surrounding, for instance, an HII region. For this survey we favor the outflow shock scenario because of the high $\mathrm{SiO}$ detection rates in the HMPOs which have yet to form HII regions or create PDRs. We note that the observations of Maxia et al. (2001) suggest an outflow as the origin of the $\mathrm{SiO}$ enhancement in G31.41 and G29.96, which are also included in this study

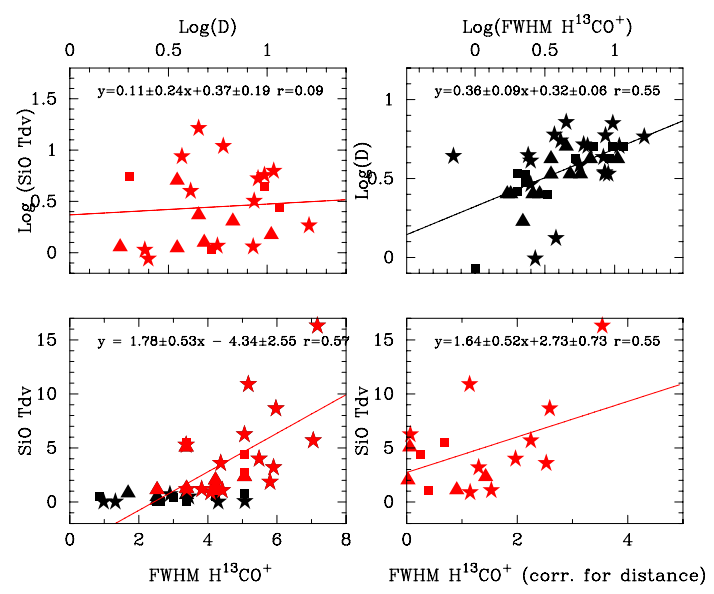

Fig. 5. (Bottom left) $\mathrm{SiO}$ integrated intensity vs. $\mathrm{H}^{13} \mathrm{CO}^{+} \mathrm{FWHM}$ and distance. Black symbols in this plot show $\mathrm{SiO}$ nondetections, red points indicate detections. The triangles $(\mathbf{\Lambda})$ indicate HMPOs, the filled squares $(\boldsymbol{\square})$ indicate HCHII regions, and the filled stars $(\star)$ indicate UCHII regions. The top panels show these two quantities as a function of source distance. The bottom righthand panel shows the $\mathrm{SiO}$ integrated intensity vs. $\mathrm{H}^{13} \mathrm{CO}^{+}$FWHM corrected for the size-linewidth relationship.

As shown in Fig. 2, the fraction of sources in each stage with active outflow motions changes. Unpublished $\mathrm{SiO}(J=$ 2-1) data from the IRAM $30 \mathrm{~m}$ taken simultaneously with the $\mathrm{CO}$ data of Beuther et al. (2002b) of the HMPOs in this sample show detections in $\mathrm{SiO}(J=2-1)$ for $11 / 12$ of the HMPOs in our study. This is a $92 \%$ detection rate (at a velocity resolution of $\sim 3.45 \mathrm{~km} \mathrm{~s}^{-1}$, and an rms noise limit of approximately $0.03 \mathrm{~K}$ ), whereas at the $J=8-7$ transition, our detection rate is slightly lower at $58 \%$. Gusdorf et al. (2008) calculate relative intensities of various rotational transitions of $\mathrm{SiO}$, and find that the 2-1 transition is brighter than the $8-7$ transition when the shock velocity is less than $30 \mathrm{~km} \mathrm{~s}^{-1}$. They also point out that for high density $\left(n \sim 10^{6} \mathrm{~cm}^{-1}\right)$, shocks with velocities greater than $\sim 32 \mathrm{~km} \mathrm{~s}^{-1}$ become dissociative $J$-shocks. As high-mass star formation tends to happen in regions with densities this high, we suggest that shock velocities of $\sim 27 \mathrm{~km} \mathrm{~s}^{-1}$ could account for our lowered $\mathrm{SiO} J=8-7$ detection rate in our sources, regardless of evolutionary stage.

It is unclear why the detection rate of $\mathrm{SiO}$ drops below $50 \%$ for the HCHII regions when, for both HMPOs and UCHII regions, the detection rates are much higher. The integrated intensities of the $\mathrm{SiO}$ for $\mathrm{HCHII}$ regions with detections correlate very well with those found for the other stages. In fact, this is our tightest correlation in Fig. 3.

The bottom lefthand panel of Fig. 5 shows the integrated intensity of $\mathrm{SiO}$ plotted against the full width at half maximum (FWHM) of the $\mathrm{H}^{13} \mathrm{CO}^{+}$line. Along with this, we plot the logarithms of these values against the log of the distances to the sources. We note that the intensity of the $\mathrm{SiO}$ line does not correlate with distance to source (correlation coefficient $=0.09$ ), however the FWHM of the $\mathrm{H}^{13} \mathrm{CO}^{+}$does (correlation coefficient $=0.55$ ). Since the observing beam is the same for each $\mathrm{H}^{13} \mathrm{CO}^{+}$observation, increased distance to source corresponds to a larger observed area. The correlation in the top righthand panel of Fig. 5 is approximately the Larson size-linewidth relation (Larson 1981). The bottom righthand panel of Fig. 5 shows a plot of $\mathrm{SiO}$ integrated intensity vs. the FWHM of $\mathrm{H}^{13} \mathrm{CO}^{+}$ corrected for the equation given in the top righthand panel (corrected for the size-linewidth relation). The correlation between the integrated intensity of $\mathrm{SiO}$ and linewidth remains. Many of 
the line widths are still much greater than thermal as well, suggesting that it is easier to detect $\mathrm{SiO}$ in more turbulent regions. It is not clear whether this relationship is caused by, or a product of, the shocks responsible for the $\mathrm{SiO}$ intensity: it is the integrated intensity of the $\mathrm{SiO}$ that scales with the FWHM, not just the detections of $\mathrm{SiO}$.

\section{Discussion and conclusions}

We have noted the role beam dilution may play in our nondetections; however, our results are, in general, consistent with previous studies that have either looked at the infall or outflow signatures in high-mass star-forming regions at different evolutionary stages. Our results are consistent with the region undergoing large-scale infall that grows with time.

Interestingly, towards IRAS20126+4107 we do not detect $\mathrm{SiO}$, yet there is a well known $\mathrm{SiO}$ jet coming from this source (Cesaroni et al. 1999). The intensity and size of this jet are small enough that we would expect it to be too beam-diluted to be detected in our current survey. However, our infall detections are consistent with the the high-resolution observations of Beltrán et al. (2011) for G10.6, G19.61, and G29.96. We and they detect infall signatures in the first two sources, but not in the third. Their infall velocities, and mass infall rates are much higher than ours, owing to different methods for determining the infall velocity (two-layer infall modeling vs. peak velocity shifting). Maxia et al. (2001) suggest that large-scale infall is occurring in both G29.96 and G31.41. The latter is consistent with our results, the former is not.

Our outflow detection rates appear to be at odds with the $\mathrm{SiO}$ results of López-Sepulcre et al. (2011), but this discrepancy appears to be due to our sensitivity limits. In the UCHII region stage, there are no sources with infall signatures that do not show evidence of outflows. This suggests that if a source is still infalling at this late stage, it is likely that it is still powering a large outflow. The older sources with $\mathrm{SiO}$ outflow signatures and no infall signatures may be showing the remnants of previous infall activity, since $\mathrm{SiO}$ only stays in the gas phase for about $10^{4} \mathrm{yr}$. Our results are consistent with the region undergoing bulk infall that grows with time.

Although we see no clear infall vs. outflow ratio with age, we can say from our survey that, as a high-mass star-forming region ages, the likelihood of observing both infall and outflow together increases (black line in Fig. 2), and the intensity of the $\mathrm{SiO}$ that is detected also increases with the age of the outflow.

Acknowledgements. The authors would like to thank Remo Tilanus for his help in reducing the data, and the anonymous referee and the editor for their insights that sharpened the arguments presented in this manuscript. L.T. acknowledges financial support from ASI under contract with INAF-Osservatorio Astrofisico di Arcetri.

\section{References}

André, P., Ward-Thompson, D., \& Barsony, M. 1993, ApJ, 406, 122 Arce, H. G., \& Sargent, A. I. 2004, ApJ, 612, 342

Beltrán, M. T., Cesaroni, R., Neri, R., \& Codella, C. 2011, A\&A, 525, A151
Beuther, H., Schilke, P., Menten, K. M., et al. 2002a, ApJ, 566, 945 Beuther, H., Schilke, P., Sridharan, T. K., et al. 2002b, A\&A, 383, 892 Beuther, H., Churchwell, E. B., McKee, C. F., \& Tan, J. C. 2007, Protostars and Planets V, 165

Bontemps, S., Andre, P., Terebey, S., \& Cabrit, S. 1996, A\&A, 311, 858

Caselli, P., Hartquist, T. W., \& Havnes, O. 1997, A\&A, 322, 296

Cesaroni, R., Felli, M., Jenness, T., et al. 1999, A\&A, 345, 949

Churchwell, E., Sievers, A., \& Thum, C. 2010, A\&A, 513, A9

Crimier, N., Ceccarelli, C., Alonso-Albi, T., et al. 2010, A\&A, 516, A102

De Vries, C. H., \& Myers, P. C. 2005, ApJ, 620, 800

Di Francesco, J., Myers, P. C., Wilner, D. J., Ohashi, N., \& Mardones, D. 2001, ApJ, 562, 770

Egan, M. P., Shipman, R. F., Price, S. D., et al. 1998, ApJ, 494, L199

Enoch, M. L., Evans, N. J., Sargent, A. I., \& Glenn, J. 2009, ApJ, 692, 973

Faúndez, S., Bronfman, L., Garay, G., et al. 2004, A\&A, 426, 97

Fuller, G. A., Williams, S. J., \& Sridharan, T. K. 2005, A\&A, 442, 949

Furuya, R. S., Cesaroni, R., \& Shinnaga, H. 2011, A\&A, 525, A72

Gregersen, E. M., Evans, I., Zhou, S., \& Choi, M. 1997, ApJ, 484, 256

Gusdorf, A., Cabrit, S., Flower, D. R., \& Forêts, G. P. D. 2008, A\&A, 482, 809

Hill, T., Burton, M. G., Minier, V., et al. 2005, MNRAS, 363, 405

Hofner, P., \& Churchwell, E. 1996, A\&AS, 120, 283

Hunter, T. R. 1997, Ph.D. Thesis

Keto, E. 2007, ApJ, 666, 976

Klaassen, P. D., \& Wilson, C. D. 2007, ApJ, 663, 1092

Klaassen, P. D., \& Wilson, C. D. 2008, ApJ, 684, 1273

Kurtz, S., Churchwell, E., \& Wood, D. O. S. 1994, ApJS, 91, 659

Larson, R. B. 1981, Roy. Astron. Soc., 194, 809

Leurini, S., Codella, C., Gusdorf, A., et al. 2011, A\&A, submitted

López-Sepulcre, A., Cesaroni, R., \& Walmsley, C. M. 2010, A\&A, 517, A66

López-Sepulcre, A., Walmsley, C. M., Cesaroni, R., et al. 2011, A\&A, 526, L2

Mardones, D., Myers, P. C., Tafalla, M., et al. 1997, ApJ, 489, 719

Mateen, M., Hofner, P., \& Araya, E. 2006, ApJS 167, 239

Maxia, C., Testi, L., Cesaroni, R., \& Walmsley, C. M. 2001, A\&A, 371, 287

Molinari, S., Pezzuto, S., Cesaroni, R., et al. 2008, A\&A, 481, 345

Myers, P. C., Mardones, D., Tafalla, M., Williams, J. P., \& Wilner, D. J. 1996, ApJ, 465, L133

Pineau des Forets, G., Flower, D. R., \& Chieze, J.-P. 1997, in Herbig-Haro Flows and the Birth of Stars, ed. B. Reipurth, \& C. Bertout (Kluwer Academic Publishers), IAU Symp., 182, 199

Plume, R., Jaffe, D. T., \& Evans, N. J. 1992, ApJS, 78, 505

Plume, R., Jaffe, D. T., Evans, I., Martin-Pintado, J., \& Gomez-Gonzalez, J. 1997, ApJ, 476, 730

Purcell, C. R., Balasubramanyam, R., Burton, M. G., et al. 2006, MNRAS, 367, 553

Rathborne, J. M., Jackson, J. M., \& Simon, R. 2006, ApJ, 641, 389

Reiter, M., Shirley, Y. L., Wu, J., et al. 2011, ApJ, 74040

Schilke, P., Walmsley, C. M., des Forets, G. P., \& Flower, D. R. 1997, A\&A, 321, 293

Shirley, Y. L., Evans, I., Young, K. E., Knez, C., \& Jaffe, D. T. 2003, ApJS, 149, 375

Shu, F. H., Adams, F. C., \& Lizano, S. 1987, ARA\&A, 25, 23

Spezzi, L., Alcalá, J. M., Covino, E., et al. 2008, ApJ, 680, 1295

Sridharan, T. K., Beuther, H., Schilke, P., Menten, K. M., \& Wyrowski, F. 2002, ApJ, 566, 931

Sun, Y., \& Gao, Y. 2009, MNRAS, 392, 170

Tsamis, Y. G., Rawlings, J. M. C., Yates, J. A., \& Viti, S. 2008, MNRAS, 388, 898

Walsh, A. J., Hyland, A. R., Robinson, G., \& Burton, M. G. 1997, MNRAS, 291, 261

Walsh, A. J., Burton, M. G., Hyland, A. R., \& Robinson, G. 1998, MNRAS, 301, 640

Wood, D. O. S., \& Churchwell, E. 1989, ApJS, 69, 831

Wu, J., \& Evans, N. J. 2003, ApJ, 592, L79

Wu, J., Evans, N. J., Shirley, Y. L., \& Knez, C. 2010, ApJS, 188, 313

Zapata, L. A., Palau, A., Ho, P. T. P., et al. 2008, A\&A, 479, L25

Zapata, L. A., Tang, Y.-W., \& Leurini, S. 2010, ApJ, 725, 1091

Zinnecker, H., \& Yorke, H. W. 2007, ARA\&A, 45, 481 